\title{
A Case of Perianal Necrotizing Fasciitis from Perianal Abscess with Integrated Chinese and Western Medicine Treatment
}

\author{
Shenghua Du1, Jingtao Sha2*, Xiangyan Yan1, Peizheng Han1 \\ ${ }^{1}$ Shaanxi University of Chinese Medicine, Xianyang 712000, Shaanxi Province, China \\ ${ }^{2}$ Xi'an Hospital of Traditional Chinese Medicine, Xi'an 710000, Shaanxi Province, China \\ *Corresponding author: Jingtao Sha, 1245328255@qq.com
}

\begin{abstract}
This article discusses a case of perianal necrotizing fasciitis from perianal abscess with integrated Chinese and western medicine treatment. It has been concluded that the treatment of perianal necrotizing fasciitis from perianal abscess should focus on dispelling evil, tonifying deficiency, or attack and supplement at the same time in order to achieve the purpose of dispelling evil without harming the right while supporting the right without leaving evil.
\end{abstract}

Keywords: Necrotizing fasciitis; Infection; Sepsis

Publication date: September 2021; Online publication: September 30, 2021

\section{Introduction}

Necrotizing fasciitis is an acute and critical disease under the category of anorectal diseases. It is a severe infectious disease caused by multiple bacteria (including aerobic and anaerobic bacteria) around the anus and urogenital triangle ${ }^{[1]}$. The disease is characterized by a large-scale progressive necrosis of the dermis, subcutaneous tissue, and fascia without involving the muscles. Its onset and progress are rapid. In addition, the infection easily spreads to the perineum, abdomen, and even the whole body, resulting in complications such as sepsis, septic shock, and multiple organ failure (MODS). The death rate of the disease is as high as $9 \%$ to $25 \%$ or even higher. This article discusses a case of perianal necrotizing fasciitis from perianal abscess with integrated Chinese and Western medicine treatment.

\section{Case analysis}

Patient H, male, 28 years old, was admitted to the emergency department at 0953 hour on November 30, 2019, with perineal and abdominal swelling as well as pain after a perianal abscess surgery. The admission examination (lithotomy position) by a specialist was as follows: upon visual examination, the surgical incision was not healed at 3 and 5 points around the anus with a size of about $10 \mathrm{~cm} \times 8 \mathrm{~cm}$ and $6 \mathrm{~cm}$ deep with foul, turbid, and blackened exudate as well as necrotic tissue at the edge; the skin around the anus and perineum was dark red, the skin out of the scrotum was broken, and there was fetid exudate; on his left side, there was redness spreading from bottom to the chest, and on his right side, the redness spread from bottom to the umbilicus; digital rectal examination revealed that the edge of the anal incision was soft and tender, the penis was edematous, scrotum was swollen, and purulent ulceration was noted; the mass at the abdomen and right anterior chest was firm, tender, and twisted under the skin. 
Upon admission, the investigation results were as follows: white blood cell (WBC) was $14.0 \times 10^{9} / \mathrm{L}$ $(\uparrow)$, red blood cell (RBC) was $3.18 \times 10^{12} / \mathrm{L}(\downarrow)$ ), hemoglobin (HGB) was $115 \mathrm{~g} / \mathrm{L}(\downarrow)$, neutrophils percentage (NEUT) was $92.20 \%(\downarrow)$, absolute count of neutrophils (NEUT\#) was $12.95 \times 10^{9} / \mathrm{L}(\uparrow)$ ), lymphocytes percentage (LYM\%) was $4.70 \%(\downarrow)$, absolute count of lymphocytes (LYM\#) was $0.66 \times 10^{9} / \mathrm{L}(\downarrow)$ ), and platelets were within normal range; international normalized ratio (INR) was $1.42(\uparrow)$, thrombin time (TT) was 16.0 seconds $(\uparrow)$, prothrombin time activity percentage (PT \%) was $49.40 \%(\downarrow)$, fibrin degradation product (FDP) was $6.5 \mathrm{ug} / \mathrm{ml}(\uparrow)$, potassium $(\mathrm{K})$ was $3.4 \mathrm{mmol} / \mathrm{L}(\downarrow)$, iron $(\mathrm{Fe})$ was $5.6 \mathrm{mmol} / \mathrm{L}(\downarrow)$, calcium (CA) was $1.81 \mathrm{mmol} / \mathrm{L}(\downarrow)$, urea was $123 \mathrm{mmol} / \mathrm{L}(\downarrow)$, procalcitonin (PCT) was $1.89 \mathrm{ng} / \mathrm{ml}(\uparrow)$. The nine items of blood transfusion were negative, and ECG showed sinus tachycardia.

Diagnosis upon admission: (1) necrotizing fasciitis; (2) sepsis; (3) electrolyte disorder. After excluding the contraindications, the debridement of the necrotizing tissue was performed in the operating theatre on the November 30 . The events of the surgery were as follows: after general anesthesia, the lithotomy position was routinely disinfected; a large amount number of purulent discharge was noted from the original surgical incision at the back of the anus; on finger diagnosis, the wound reached deep to the pelvic rectal space, and the abscess cavity spread to the scrotum on both sides and upward, with necrosis and ulceration on the scrotum surface, redness over the abdomen and the right chest wall, as well as swollen and twisted mass; the original surgical incision was expanded, the infected necrotic tissue was removed until there were no fresh infected fasciae, it was gradually removed upward along the necrotic part to the scrotum, bilateral testicles were protected, and subcutaneous fat was separated from the fascia layer; at the same time, the incision in the hip was increased and a rubber band was hung between each incision to promote smooth drainage; necrotizing fascia and adipose tissue were gradually removed from the left inguinal mass up to the left anterior chest, and the surgical method of skipping relay incision and short-cut drainage was used. The right side was treated in the same way. The skin margin was trimmed, the bleeding was fully stopped, the wound was cleaned with hydrogen peroxide as well as normal saline, and it was wrapped with oil gauze. Considering the large area of infection, affecting the waist, back, buttocks, perineum, chest, and abdomen, as well as the possible life-threatening situations, such as septic shock, nausea, arrhythmias, respiratory arrest, cardiac arrest, electrolyte disorders, and multiple organ dysfunction syndrome, the patient was transferred to ICU after the surgery.

Diagnosis upon transferring to ICU (2229 hour on November 30, 2019): (1) necrotizing fasciitis; (2) sepsis; (3) electrolyte disorder - hypokalemia and hypocalcemia; (5) abnormal liver function; (6) hypoproteinemia; (7) anemia. In consideration of the serious infection and the pending etiological results, meropenem and linezolid were administered. The antibiotic regimen was adjusted after the etiological results were out.

At 2220 hour on November 30, 2019, the patient's serum albumin was measured at $22.8 \mathrm{~g} / \mathrm{L}$ and $20 \%$ human albumin was infused.

At 1044 hour on December 1, 2019, the patient regained consciousness but still had fever; his maximum body temperature was $38^{\circ} \mathrm{C}$. The 12 -hour in and out volume was as follows: the total intake was $3295 \mathrm{ml}$ and total output was $1350 \mathrm{ml}$ (all urine output). The blood investigations were as follows: magnesium $(\mathrm{Mg})$ was $1.1 \mathrm{mmol} / \mathrm{L}(\uparrow)$, Fe was $5.3 \mathrm{mmol} / \mathrm{L}(\downarrow)$, CA was $1.77 \mathrm{mmol} / \mathrm{L}(\downarrow)$, D-dimer (D-D) was $2.6 \mathrm{ug} / \mathrm{ml}(\uparrow)$, activated partial thromboplastin clotting time (APTT) was 37.6 seconds ( $\uparrow$ ), INR was $1.51(\uparrow)$, TT was 18.1 seconds $(\uparrow)$, PT- $\%$ was $44.9(\downarrow)$, FDP was $7.0 \mathrm{ug} / \mathrm{ml}(\uparrow)$; PCT was $2.46 \mathrm{ng} / \mathrm{ml}(\uparrow)$, WBC was $15.1 \times 10^{9} / \mathrm{L}(\uparrow)$, RBC was $3.26 \times 10^{12} / \mathrm{L}(\downarrow)$, NEUT\% was $86.20 \%(\downarrow)$, LYM\% was $8.80 \%(\downarrow)$, C-reactive protein was $54.52 \mathrm{mg} / \mathrm{L}(\uparrow)$, total protein $(\mathrm{Tp})$ was $19.50 \mathrm{~g} / \mathrm{L}(\downarrow)$, ALT was $51 \mathrm{u} / \mathrm{L}(\uparrow)$, AST was $47 \mathrm{u} / \mathrm{L}(\uparrow)$, direct bilirubin (DBL) was $19.3 \mathrm{umol} / \mathrm{L}$, total bilirubin (TBL) was $24.5 \mathrm{umol} / \mathrm{L}, \mathrm{Tp} 39.7 \mathrm{~g} / \mathrm{L}$. Gastric occult blood test was positive. The patient's infection index, body temperature, and exudate were high. Meropenem and linezolid were administered continuously to fight infection. Pantoprazole, vitamin 
K1, and Kangfuxin solution were given to inhibit acid, protect the gastric mucosa, and supplement coagulation factors. Necessary blood transfusion was done based on the hemoglobin levels. The doctor debrided and changed the dressing of the wound as well as applied Xiaozhong Cuyu decoction to reduce the inflammation. The prescription was as follows: 30 grams of Herba Portulacae, 15 grams of Cacumen Platycladi, 15 grams of Rhizoma Atractylodis, 15 grams of Radix Saposhnikoviae, 15 grams of Fructus Aurantii, 30 grams of Rhizoma smilacis glabrae, 20 grams of Cortex Phellodendri, 30 grams of Herba Taraxaci, 12 grams fo Rhizoma Bletilla, 12 grams of Rhizoma Corydalis, and 12 grams of Radix Sanguisorbae.

At 0957 hour on December 2, the patient's vital signs were relatively stable, and his body temperature was lower than before. His PCT decreased but the ratio of leukocytes to neutrophils was still high. The treatment with meropenem and linezolid continued along with the dressing and the application of Xiaozhong Cuyu decoction.

On the evening of December 3, it was found that Escherichia coli was positive in the pus culture, and it was sensitive to meropenem and imipenem. Linezolid was stopped and the measures of multidrug resistance prevention and isolation were strictly implemented. The patient's body temperature reduced, and the infection indexes, such as hemogram and PCT, decreased. Vitamin K1 was stopped, human albumin was continued to be supplemented, and reduced glutathione was injected to protect the liver and lower transaminases.

On December 4, debridement of the necrotizing tissue was performed again as follows: after general anesthesia, in the lithotomy position, the skin outside the wounds on both sides of the abdomen was red and swollen, and the infection had spread near the axillary midline; the rubber band was removed between each incision, the necrotic tissue was completely removed from the wound, the wound was repeatedly washed with hydrogen peroxide and normal saline, the normal skin around the wound was cleaned, the wound at the abdominal wall and chest wall was sutured, vacuum sealing drainage (VSD) dressing was placed over the wound according to the size of the wound, the wound was covered with Iodoform gauze, the semi permeable membrane was placed, and the drainage pipe was connected to the negative pressure device. After the surgery, hemoglobin was $88 \mathrm{~g} / \mathrm{L}(\downarrow)$. In consideration of anemia caused by intraoperative bleeding and infection, 2 units of red blood cell suspension were infused to correct the deficiency. The rest of the treatment was the same as before.

On December 5, 2019, the patient had venous plexus thrombosis of the left lower limb and was treated with low molecular weight heparin. A drainage tube was placed in the affected area and connected to a negative pressure; drainage and suction was continued with saline. At 2120 hour, hemoglobin was still 88 $\mathrm{g} / \mathrm{L}(\downarrow)$, and 2 units of erythrocyte suspension were infused.

At 1005 hour on December 6, 2019, the patient suddenly developed shivers and high fever along with chest and abdominal pain; his heart rate was 128 beats per minute, breathing rate was 42 breaths per minute, invasive blood pressure was $178 / 95 \mathrm{mmHg}$, oxygen saturation was $88 \%$, body temperature was $38.7^{\circ} \mathrm{C}$, and harsh breath sounds were heard in both lungs with reduced breath sounds in both lower lungs. The doctors in the ICU immediately arranged for rescue and contacted the anorectal department as well as the surgeons to change the wound dressing and evaluate the condition. In view of the high fever and chills, the possibility of an aggravation of infection and blood inflow were considered; thus, linezolid was added. Samples for blood culture, secretion culture, and urine culture were taken, and the patient was given dexamethasone for support. Blood gas showed that his oxygenation index was 113 with high potassium and low sodium. Considering acute respiratory distress syndrome (ARDS) and intracellular potassium shift, sodium ion supplement and calcium were given to protect the cardiomyocytes. Color ultrasound revealed bilateral pleural effusion and edema over both lower limbs. Albumin and furosemide were given to reduce the swelling while maintaining the acid-base balance of water and electrolytes. The patient was also given 
oral Chinese decoction, mainly for clearing heat and detoxification, nourishing Yin and blood, promoting blood circulation, as well as cooling the blood. The specific prescription was as follows: 20 grams of Radix Astragali seu Hedysari, 2 grams of Radix Rehmanniae Preparata, 20 grams of Radix Rehmanniae Recens, 15 grams of Radix Scrophulariae, 20 grams of Radix Angelicae Sinensis, 10 grams of Radix Trichosanthis, 20 grams of Bulbus Fritillariae Thunbergii and Herba Taraxaci, 10 grams of vinegar frankincense, 10 grams of vinegar myrrh, 20 grams of fried Rhizoma Atractylodis Macrocephalae, 20 grams of Poria cocos, 30 grams of Flos Lonicerae, 20 grams of Fructus Forsythiae, 12 grams of Platycodon grandiflorum, 12 grams of Radix Paeoniae Rubra, 10 grams of roasted Radix Glycyrrhizae, and 15 grams of Radix Pseudostellariae.

On December 7, the patient's pus culture was positive for extended spectrum beta-lactamase Escherichia coli, and his blood culture was positive for gram-negative bacteria. Levofloxacin was added to meropenem and linezolid.

From December 8 to December 11, 2019, the patient's condition gradually stabilized, and the infection index decreased. During that period, the patient had poor appetite; thus, he was given oral mosapride to promote gastrointestinal motility in addition to the decoction of four-drug juice and rhubarb powder to activate Qi and dredge the inner organs. Then he was transferred to the anorectal department for further treatment. The anorectal department continued the anti-infectives and regularly monitored various indexes. In the early stage, the medical prescription was changed to twice a day, mainly with detoxification ointment and detumescence ointment to promote wound drainage and reduce inflammation. In the later stage, Jiuhua ointment was used to promote the growth of granulation tissue and accelerate wound healing.

On December 30, the patient was discharged in a stable condition.

\section{Conclusion}

Once perianal necrotizing fasciitis is diagnosed clinically, surgical debridement must be carried out immediately, supplemented by effective anti-infectives, nutritional support, and symptomatic treatment. Although perianal necrotizing fasciitis is caused by external toxins and evil excess, it is actually due to the internal deficiency of healthy Qi, which is very easy to turn yellow or sink in. In the process of treatment, it is necessary to weigh the rise and fall of good and evil, focus on dispelling evil, tonifying deficiency, or attack and supplement at the same time so as to achieve the purpose of dispelling evil without harming the right, and supporting the right without leaving evil ${ }^{[2]}$. In view of traditional Chinese medicine, Xiaozhong Cuyu decoction was used in this case to reduce swelling, promote healing, and reduce local inflammatory reaction; traditional Chinese medicine was also taken orally to clear heat, detoxify, nourish Yin and blood, as well as to activate and cool the blood, resulting in excellent outcomes.

\section{Disclosure statement}

The authors declare that there is no conflict of interest.

\section{References}

[1] Song JZ, Wei SX, Li Q, et al., 2021, Observation on the Efficacy of Integrated Traditional Chinese and Western Medicine in the Phased Treatment of Perianal Necrotizing Fasciitis. Modern Journal of Integrated Traditional Chinese and Western Medicine, 30(10): 1056-1060.

[2] Liu RR, Cao YQ, Yao YB, 2020, Progress in the Treatment of Perianal Necrotizing Fasciitis with Traditional Chinese and Western Medicine. Chinese Journal of Surgery of Integrated Traditional and Western Medicine, 26(02): 382-385. 